\title{
Comparative Study of Image Retrieval using Shape as Prominent Feature
}

\author{
Anita Kinnikar \\ Student, Dept. of CSE, \\ BVBCET, Hubli, Karnataka, India \\ Padmashree Desai \\ Assoc. professor, Dept. of CSE, \\ BVBCET, Hubli, Karnataka, India
}

\begin{abstract}
The process of retrieving relevant images from an expansive accumulation is broadly utilized as a part of uses of computer vision. So as to enhance the retrieval effectiveness, a efficient and accurate framework is required. Retrieving the similar images with the help of image features such as shape, color and texture is referred as content based image retrieval (CBIR). It is extracted and represented in the form of feature vector. The images have the minimum distance between their feature vectors, if they are most similar. This paper provides the correlation of various CBIR frameworks and similarity measures are used to find the similarity between two images. We are proposing new framework for image retrieval using wavelet decomposition and Local Binary Patterns (LBP) using shape as prominent feature by identifying issues in existing CBIR systems.
\end{abstract}

Keywords - CBIR, feature vector, similarity measures, wavelet decomposition, local binary patterns.

\section{INTRODUCTION}

With the advancement of Internet and the accessibility of effective image capturing gadgets, for example, image scanners, digital cameras and high capacity open systems, shabby capacity; the measure of advanced image gathering is expanding quickly. There is need of image seeking, retrieval and browsing apparatus by the clients from different areas including design, remote detecting, wrongdoing aversion, distributed, engineering, solution and so forth. We can fulfill this above said assignment with the assistance of numerous broadly useful image retrieval frameworks. Basically they are divided into text based image retrieval and Content based image retrieval.

In early image retrieval systems, the images or multimedia were manually annotated with text and textual queries were used as the basis for image retrieval or multimedia. These text strings can either be related to a feature in the image itself, or to the image (e.g., place, object name, data of incidence etc.). These strings are stored and can be sought structuredly, as in structured query language (SQL). Yet, there were critical issues which are because of the high price of manual text annotation for huge collections and also the lack of information that can be captured easily in the text. The difficulties of indexing, matching and retrieving image information have led to the development of various techniques of image retrieval.

Image retrieval is the utilization of computer vision techniques to search digital images in large databases. During the last several years, CBIR emerged as powerful tool to efficiently retrieve the images which are visually similar to the query image. The basic idea behind this approach is the representation of image as a feature vector and to measure the similarities between the images with the distance between their corresponding feature vectors according to some metrics. The finding of correct features to represent the images with, as well as the similarity metric that groups visually similar images together, are the important milestone in construction of any CBIR system. In Content based image retrieval, the images are indexed by their visual contents, such as shape, color, texture, motion, structure and combination of these.

The CBIR framework shown in Fig.1 extracts visual features such as shape, texture, color and spatial information of each image and stores as a feature vector of the database. The feature information of every image is smaller in size compared with the image information. While retrieving similar images from database, framework extracts visual 
features such as shape, texture, color and spatial information. Next it compares feature vector of query image with feature vectors of database using similarity measures. The image having least distance is the most similar to the query image.

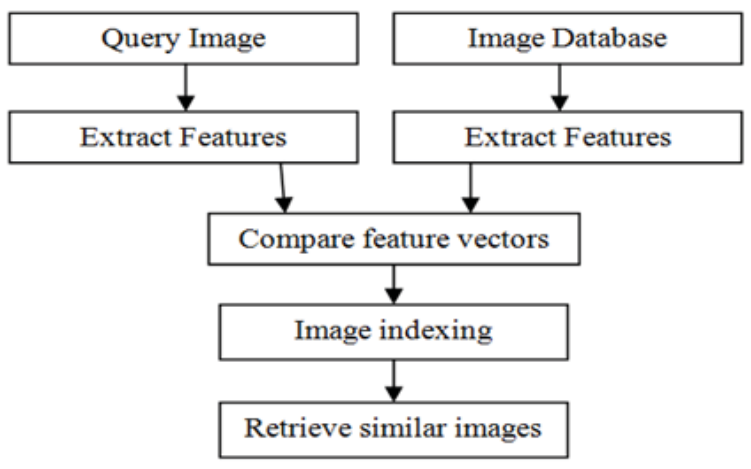

Fig 1. Structure of CBIR framework

\section{RELATED WORK}

Amol P Bhagat and Mohammad Atique [1], proposed a new framework which combines shape, color and texture features. Color moments, local color histogram and global color histogram techniques are used for color feature extraction and co-occurrence method is used for extracting texture features from image. The image segmentation and geometric moment are used for shape analysis. The co-occurrence method is found to be more precise for all image classes as compared to other methods.

P. S. Hiremath and Jagadeesh Pujari [2], proposed a new technique that uses multi-grid framework for image retrieval using shape, color and texture features. The images are segmented into non-overlapping tiles. From each tiles at two different resolutions texture and color features are extracted in two grid framework. Color moments are used to extract color features, texture features are extracted using gabor filter and gradient vector flow fields are used to extract shape features. Invariant moments are used to represent the shape features. Image similarity is based on an integrated matching scheme. Combination of these shape, texture and color features provides a combined feature vector for image retrieval.

N. Kavitha and P. Jeyanthi [3], proposed a Content Based Image Retrieval technique based on image shape, texture and color features. Here, the color histogram features are analyzed based on HSV color space which is used effectively to describe the color features. The shape of image is analyzed by efficient Polar Raster Edge Sampling technique which provides exemplary feature extraction method by binning the counts of the edge points in radial and angular directions to store the feature vectors. The texture is analyzed using FDCT based haralick features which provides high level detailed texture information. Curvlet used to represent the texture information of an image at different pixel positions and it is given significant details for better feature extraction. The similarity measures had done between feature vectors of input images and database images using Euclidean distance. Here, genetic coding is used to fuse similarity scores to select the optimal weight feature vector.

Jiayin Kang and Wenjuan Zhang [4], proposed a technique for content-based image retrieval by combining the shape color and texture features. The proposed method extracts color histogram to form feature vector in HSI color space. Extracted texture feature by using gray co-occurrence matrix and extracted shape feature by using zernike moments. Finally, it combined the color, shape and texture features to form the combined feature vectors of image.

Swati Agarwal and et al. [5] proposed a method for image retrieval using Edge Histogram Descriptor and Discrete Wavelet Transform. The proposed method is based on shape, texture and color features. The query image is decomposed into wavelet coefficients. The wavelet coefficients give vertical, diagonal and horizontal features of the image. After wavelet transform, the Edge Histogram Descriptor is used on selected wavelet coefficients to collect the information about the dominant edge orientations. Combination of DWT and EHD methods increases the retrieval performance of image retrieval system.

Savita Gandhani and et al .[6], proposed a novel approach in content-based image retrieval (CBIR) by combining the low level features such as shape, color and texture features. Here at first, transformed the color space from RGB model to HSV model, and then extracted color histogram to form color feature vector. Next, extracted the texture feature by using Block Difference of Inverse Probabilities (BDIP) and Block-Based Local Correlation (BVLC) 
moment and At last, applied canny edge detection to extract the shape features. Finally, combined the shape, color and texture features to form the feature vectors of the entire image.

$\mathrm{Jia} \mathrm{Li}$ and et al.[11], proposed a Content based image retrieval using region segmentation. Proposed strategy utilizes IRM (Integrated Region Matching), a new similarity measure for region based image likeness correlation. The focused on image retrieval frameworks speak to a image by an arrangement of regions, generally comparing to items, which are portrayed by elements reflecting color, texture, shape, and location properties. The IRM measure for assessing general similarity between images fuses properties of the considerable number of districts in the images by a region matching scheme. Contrasted and recovery in view of individual regions, the general similarity approach decreases the impact of off segmentation, clears up the semantics of a specific district, and empowers a straightforward querying interface for region based image retrieval frameworks. The application to a database of around 200,000 universally useful images indicates outstanding robustness to image changes, for example, intensity variety, sharpness variety, color mutilations, shape distortions, cropping, shifting, and rotation.

Manisha Lumb and Poonam Sethi [13], proposed a method, in which the relevant images are retrieved using Wavelet Decomposition and GLCM techniques. The proposed method is applied on different types of images, among them the HSV images are retrieved with better efficiency with compared to others by utilizing both methods.

Zhujun Wang and Guangwen Zhang [14], proposed a content base image retrieval technology. Here author selected the specific gallery - cheongsam library to study. Based on texture feature of cheongsam library, proposed method uses two kinds of texture retrieval methods - wavelet transform and LBP.

Padmashree Desai and et al. [17], proposed a framework that extracts shape feature of the query image using wavelet decomposition for retrieving the similar images from the database. The proposed technique identifies the edges by decomposing the image into four segments. Distinctive pixel data accessible on approximation, horizontal, vertical and diagonal parts are considered. Edge maps got are utilized to develop a shape feature vector. Edge location is done utilizing wavelet family, for example, Daubechies (db2) and Coiflets (coif2). Efficiency of retrieval is determined by looking at consequences of Daubechies and Coiflets. The proposed strategy is invariant to rotation, scaling and translation.

Padmashree D. Desai and et al. [18], proposed a framework that extracts shape and texture feature of the query image using multilevel wavelet decomposition for retrieving the similar images from the image dataset. The key contribution of proposed work is to detect the edges using wavelet based techniques. Two level wavelet decomposition helps to retain the different pixel information to obtain the different edge images in turn get robust shape features.

Padmashree D. Desai ant et al. [19], proposed an image retrieval method where shape of an image is extracted by applying morphological operators and wavelet decomposition. Proposed technique uses wavelet decomposition to find the edge map and to obtain better shape features of an image it uses mathematical morphological operators. The key contribution of this technique is to overcome the problems existed in classical methods of edge detection such as poor anti-attack, noise-sensitivity and not complete information of extracted edge. The method of edge detection based on mathematical morphology not only smoothen the edges and also keep the boundary edges clear.

M. Banerjee and et al. [20], proposed another image retrieval method utilizing outwardly critical features. Groups of focuses around critical curvature regions (high, medium, frail sort) are extricated to get a delegate image. Illumination, perspective invariant color features are registered from those focuses for assessing similarity between images. Relative significance of the features are assessed utilizing a fuzzy entropy based measure registered from important and unimportant arrangement of the retrieved images set apart by the clients. The execution of the framework is tried utilizing diverse arrangement of samples from universally useful image database. Robustness of the framework has likewise been indicated when the images have experienced diverse changes.

\section{ISSUED IDENTIFIED}

- Traditional canny edge detection technique is having some drawbacks. The main drawback is, still need to connect the resulting edges to extract the complete edges.

- So far Wavelet Decomposition and LBP are used for extracting texture features.

- Smoothen edges will not be obtained using existing techniques.

- Very fine grained image details will be lost. 


\section{PROPOSED METHODOLOGY}

By studying the different edge detection and shape descriptors we are proposing a new approach for image retrieval using wavelet based shape features and LBP as shown in Fig.2.

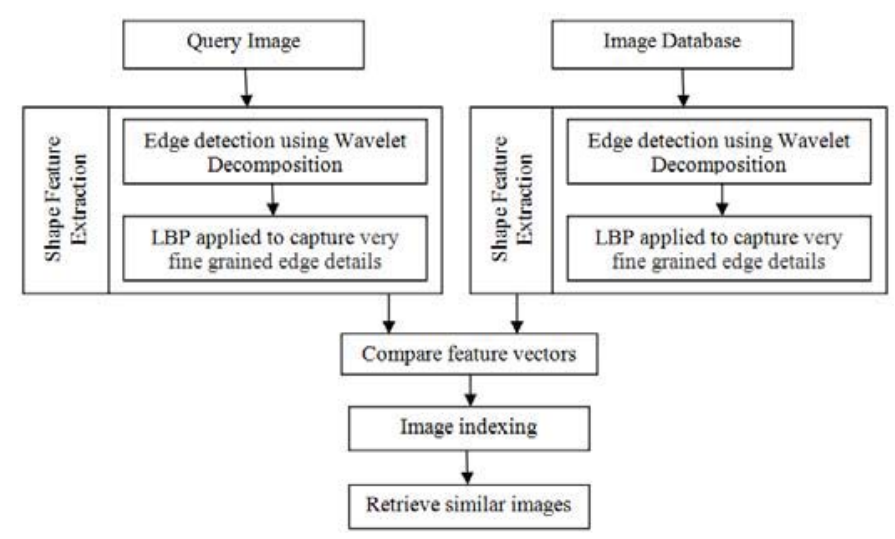

Fig.2 Image retrieval utilizing wavelet decomposition and LBP

\section{A. Feature extraction using Wavelet Decomposition}

The proposed method uses Daubechies wavelets $(\mathrm{db} 2)$ for image decomposition. Input query image is converted to gray scale image. The resulted gray scale image is decomposed into 4 components as Approximation, Horizontal, Vertical component and Diagonal component. In this technique, only alternative columns and rows of the input image are present in approximation component. Other important edge information is obtained from different components. To get all weak and strong edge information, all four components are used. Four edge maps obtained by multiplying approximation component with four masks. Moment invariants are applied on obtained edge maps to get a feature vector. The process is repeated for creating shape feature vector of Image Database.

\section{B. Feature extraction using LBP method}

The LBP Feature vector is computed as follows. First divide the examined window into cells. The method uses $3 \times 3$ mask window and threshold the window with the center pixel value. Take surrounding the neighbors of the center pixel in the vector form. For each center pixel in a cell, compare the pixel to each of its 8 neighbours in the vector form. Where the center pixel's value is greater than the neighbor's value, write "0". Otherwise, write "1". This gives an 8-digit binary number. Then the pattern should be flipped to construct directly binary number vector and Convert the binary number vector to real binary number give its decimal value. Likewise we have to get the decimal value for each cell and construct the LBP histogram to get a feature vector. The process is repeated for creating shape feature vector of Image Database.

\section{Classification}

Combine the shape feature vectors obtained by Wavelet Decomposition and LBP method. Here similarity measurement is done based on Canberra distance to retrieve similar images.

\section{CONCLUSION}

The key contribution of this paper is to provide the comparative study of different CBIR systems using shape as prominent feature and to propose a new technique for image retrieval using wavelet decomposition and LBP. This Paper lists different challenges and issues in CBIR systems. We have proposed a novel approach which uses multi resolution wavelets and LBP description to capture very fine grained details of an image to find the edges of an 
image in turn to extract the shape feature to achieve better image retrieval efficiency. Performance of the new CBIR system can be measured using precision and recall parameters.

Future work is dedicated to implement the proposed method on a benchmark image dataset and measure the performance of the CBIR system and to compare the results with existing CBIR systems.

\section{REFERENCES}

[1] Amol P Bhagat, and Mohammad Atique, "Design and Development of Systems for Image Segmentation and Content Based Image Retrieval”, in CISP Proceedings, pp.109-113, 2012.

[2] P. S. Hiremath and Jagadeesh Pujari, "Content Based Image Retrieval using Color, Texture and Shape features", in 15th International Conference on Advanced Computing and Communications, pp.780-784, 2007.

[3] N .Kavitha and P .Jeyanthi, "Exemplary Content Based Image Retrieval Using Visual Contents \& Genetic Approach" in IEEE ICCSP conference, pp. 1378-1384, 2015.

[4] Kang, Jiayin, and Wenjuan Zhang, "A framework for image retrieval with hybrid features", Control and Decision Conference (CCDC), 24th Chinese, IEEE, pp. 1326-1330, 2012.

[5] Swati Agarwal, A. K. Verma, and Preetvanti Singh, "Content Based Image Retrieval using Discrete Wavelet Transform and Edge Histogram Descriptor," in International Conference on Information Systems and Computer Networks, pp.19-23, 2013.

[6] Savita Gandhani, Rakesh Bhujade, and Amit Sinhal, "An Improved and efficient implementation of CBIR system based on combined features", in IET, pp.353-359.

[7] http://wang.ist.psu.edu/docs/related/

[8] N.Puviarasan, Dr.R.Bhavani, and A.Vasanthi, "Image retrieval using combination of texture and shape features", in International Journal of Advanced Research in Computer and Communication Engineering, Vol. 3, Issue 3, pp. 5873-5877, March 2014.

[9] M. E. ElAlami, "A novel image retrieval model based on the most relevant features", Knowledge-Based Systems, Vol. 24, pp. $23-32,2011$.

[10] Brajpal Singh Jadon, and Neelesh Gupta, "Fuzzy Logic Technique in Digital Images using Edge Detection”, in International Conference on Cloud, Big Data and Trust, Nov 13-15, RGPV, pp.192-195, 2013.

[11] J.Li, J,Z wang and G. Wiederhold, "IRM: Integrated Region Matching for Image Retrieval", in Proc.of the 8th ACM Int. Conf. on Multimedia, pp.147-156, Oct 2000. [SIMPLIcity]

[12] Y. Chen and J.Z.Wang, "A Region based Fuzzy Feature Matching Approach to Content based Image Retrieval", IEEE Trans on PAMI, vol 24, No9, pp.1252-1267, 2002. [FIRM]

[13] Manisha Lumb and Poonam Sethi, "Texture Feature Extraction of RGB, HSV, YIQ and Dithered Images using GLCM, Wavelet Decomposition Techniques", in International Journal of Computer Applications (0975 - 8887), Volume 68- No.11, pp.25-31, April 2013.

[14] Zhujun Wang and Guangwen Zhang, "Digital Image Retrieval Technology Based on Multifeature", in IEEE 2013.

[15] Y. Rubner, L.J. Guibas, and C. Tomasi, "The earth mover's distance, multi-dimensional scaling, and color based image retrieval", Proceedings of DARPA Image understanding Workshop, pp. 661-668, 1997.

[16] B.H.Shekar and Bharathi Pilar, "Shape Representation and Classification through Pattern Spectrum and Local Binary Pattern - A Decision Level Fusion Approach”, Apr 2015.

[17] Padmashree Desai, Jagadeesh Pujari, and Goudar R.H., "Image Retrieval using wavelet based shape features", in Journal of Information Systems and Communication,Volume 3, Issue 1, pp.-162-166,2012.

[18] Padmashree D.Desai, Jagadeesh Pujari, V. Kamakshi Prasad, and N.H. Ayachit, "CBIR using multilevel wavelet decomposition and adaptive thresholding techniques", in Elixir Comp. Sci. \& Engg. 62 (2013), pp.17677-17682.

[19] Padmashree D. Desai, Jagadeesh Pujari, and Nagaratna Yaligar, "Shape Based Features Extracted Using Wavelet Decomposition and Morphological Operators", in International Journal of Advanced Research in Computer Science,Volume 3, No. 3, pp.556-561,May-June 2012.

[20] M.Banerjee, M,K, Kundu, and P.K.Das, "Image Retrieval with Visually Prominent Features using Fuzzy set theoretic Evaluation”, ICVGIP 2004, India, Dec 2004. 